\title{
SIMULATION OF LOW FREQUENCY ELECTROMAGNETIC FIELD EFFECT ON CELL MEMBRANE CHANNELS MODEL
}

According to many current investigations, which approve the direct impact of potassium or sodium channels to the cell dividing processes, the presented article outlines an attempt to simulate influence of low frequency electromagnetic field to the cell membrane channels. Activation or inhibition of ion channels could be achieved by various mechanisms e.g. most common is chemical way. In contrast with this fact, the article describes simulations of external low frequency electromagnetic field application on the cell model, which should provide results comparable to the conventional methods.

Keywords: Low frequency electromagnetic field, eddy currents, potassium channels, sodium channels, cancer therapy

\section{Introduction}

From the most recent studies of human cells it is clear that bioelectrical properties of this nonlinear structure depend on many parameters complementing each other. Nowadays, most of studies are focused on transmembrane voltage and its impact on the cell cycle processes. About 40 years ago Cone et al. in their work [1] started with theories about transmembrane potential and its role in the cell dividing processes. Other studies [2,3] approved that changes in transmembrane voltage could slow down or even stop processes of cell differentiation and thus should have impact in cancer therapy.

The transmembrane voltage is influenced by concentrations of ions in extracellular and intracellular environment which depend on activation of ion channels. That means that transmembrane voltage is a complex variable which depends on various processes outside or inside the cell. Probably the most important part of this problem is activation/inhibition of ion channels, which directly influences the concentration of extra/intra cellular ions and thus has impact to the cell proliferation and differentiation.

As a proposal for solution of the said problem an attempt to simulate effect of homogenous low frequency electromagnetic field (LF EMF) exposure to the cell model extended by ion channels is presented.

\section{Hodgkin-Huxley electric cell model}

Electromagnetic field and interaction thereof with cells is best explained at the microscopic level. Each cell in a living organism is composed of two parts - the inner cytoplasm (along with the nucleus and other organelles) and the outer membrane. Conductivity-wise, the outer membrane can be considered an insulator, and as such all current seems to flow in the extracellular fluid-filled space. However, the total cell resistance is analogous to that of a simple electronic R-C circuit as can be seen in Fig. 1 - the insulating membrane being the capacitance and cytoplasm the resistance. The lipid bilayer is represented as a capacitance $\left(C_{m}\right)$. Voltage-gated and leak ion channels which represent the natural permeability of the membrane to ions are denoted by nonlinear $\left(g_{n}\right)$ and linear $\left(g_{L}\right)$ conductances, respectively. The electrochemical gradients driving the flow of ions are represented by nonlinear $\left(E_{n}\right)$, and linear $\left(E_{L}\right)$ voltage sources, ion pumps and exchangers are represented by current sources $\left(I_{p}\right)$. The resulting transmembrane voltage $\left(V_{m}\right)$ is equal to the difference between inner and external media electric potentials ( $\varphi_{i}$ and $\varphi_{e}$, respectively) [4]

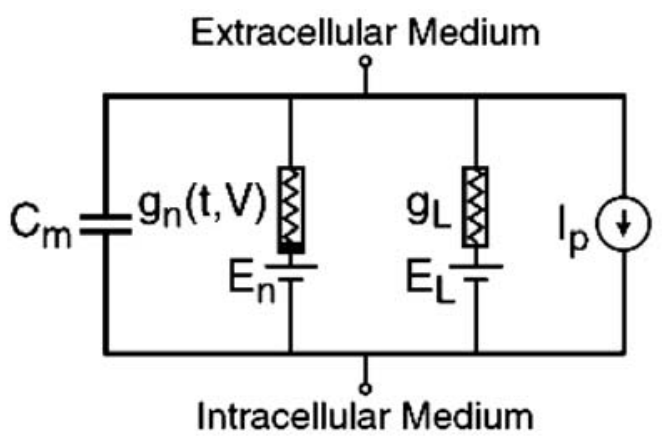

Fig. 1 Hodgkin-Huxley electric model of a biological cell [4]

The membrane current $\left(I_{m}\right)$ is comprised of two components:

- resistive (conductive) current,

- capacitive (displacement) current,

\footnotetext{
* R. Radil, J. Barabas

Department of Electromagnetic and Biomedical Engineering, Faculty of Electrical Engineering, University of Zilina, Slovakia,

E-mail: radil@fel.uniza.,sk
} 
both of which depend on the transmembrane voltage $\left(V_{m}\right)$. Thus, the membrane current can also be rewritten as a function of $V_{m}$ :

$$
I_{m} \equiv I_{m}\left(V_{m}\right)=C_{m} \times \frac{\partial V_{m}}{\partial t}+I_{p}\left(V_{m}, q\right)
$$

where the variable $q$ represents certain state variables (such as the ionic concentrations), represented by a system of differential equations. It is clear that by modifying the transmembrane voltage via application of exogenous electromagnetic fields one can directly influence cellular dynamics.

According to this template an electromagnetic model of the cell has been created as a spherical capacitor, which is shown in Fig. 2. As it is shown in the picture, this model is placed into the homogenous LF EMF generated by Helmholtz coils.

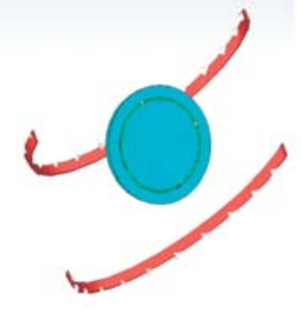

a)

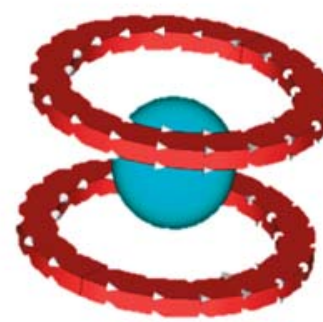

b)
Fig. 2 Cell model as a spherical capacitor: a) cross-section; b) whole model

It consists of three different layers, each of which is defined by values from Tab. 1 .

Values of material conductivities and permittivities [5]

Table 1

\begin{tabular}{|c|c|c|}
\hline & Conductivities & Relative Permittivities \\
\hline External & $\sigma_{e}=0.12 \mathrm{~S} / \mathrm{m}$ & $r_{e}=80$ \\
\hline Membrane & $\sigma_{m}=1 \mu \mathrm{S} / \mathrm{m}$ & $r_{m}=9.04$ \\
\hline Internal & $\sigma_{i}=0.53 \mathrm{~S} / \mathrm{m}$ & $r_{i}=50$ \\
\hline
\end{tabular}

\section{Modeling of low frequency electromagnetic field influence on ion channels}

In addition to the Hodgkin-Huxley model, the GoldmanHodgkin-Katz (GHK) equation from [5] is used as a basis for modeling of ion channels. Practical application of the GHK is in cell membrane physiology to determine the equilibrium potential across a cell's membrane taking into account all of the ions that are permeant through that membrane. This is necessary because perfect ion channel selectivity is not possible in living systems. The following GHK equation considers a case of various conductivities (specifically $\mathrm{Na}^{+}, \mathrm{K}^{+}$and $\mathrm{Cl}^{-}$) and is expressed as:

$$
V_{m} \beta \frac{R T}{F} \ln \frac{P_{k}\left[K^{+}\right]_{\text {out }}+P_{N a}\left[\mathrm{Na}^{+}\right]_{\text {out }}+P_{C l}\left[\mathrm{Cl}^{-}\right]_{\text {out }}}{P_{k}\left[\mathrm{~K}^{+}\right]_{m}+P_{\mathrm{Na}}\left[\mathrm{Na}^{+}\right]_{m}+P_{C l}\left[\mathrm{Cl}^{-}\right]_{m}}
$$

wherein:

$V_{m} \ldots$ is the transmembrane voltage $[\mathrm{mV}]$

$R \quad \ldots$ is the ideal gas constant $\left[8.314472 \mathrm{~J} \cdot \mathrm{K}^{-1} \cdot \mathrm{mol}^{-1}\right]$

$T \quad \ldots$ is the temperature $[\mathrm{K}]$

$F \quad \ldots$ is the Faraday's constant $\left[96485.3399{\mathrm{C} . \mathrm{mol}^{-1}}^{-1}\right.$

$P_{x} \quad \ldots$ is the concentration of a specific ions $\left[x=\mathrm{K}^{+} ; \mathrm{Na}^{+} ; \mathrm{Cl}^{-}\right]$

As it is clear from the equation and the Hodgkin-Huxley model, the ion channels represent a conductive way through the cell membrane. From this point of view the channels were modeled as a conductive tube through the membrane as it is shown in Fig. 3. Each of the channels is placed along the axes $x$ and $y$. Material properties are defined for sodium and potassium channels according to [6] and are summarized in Table 2.
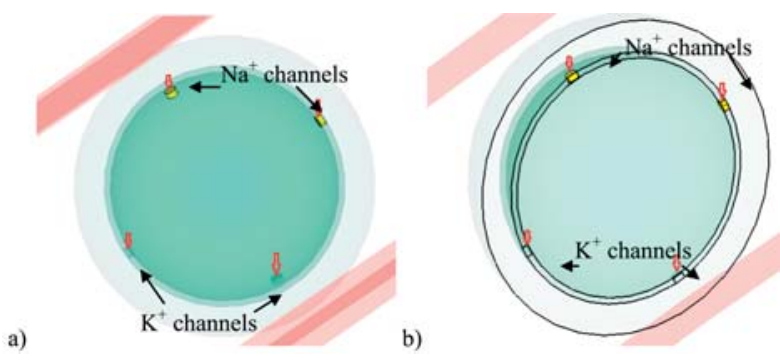

Fig. 3: Modeling of ion channels: a) localization of ion channels in the membrane; b) cross-section

Material properties of ion channels ${ }^{1)}$

Table 2

\begin{tabular}{|c|c|c|}
\hline & Sodium & Potassium \\
\hline Electric conductivity & $\sigma_{e N a}=2100 \mathrm{~S} / \mathrm{m}$ & $\sigma_{e K}=1640 \mathrm{~S} / \mathrm{m}$ \\
\hline Thermal conductivity & $\sigma_{T N a}=142 \mathrm{~W} /(\mathrm{Km})$ & $\sigma_{T K}=102.5 \mathrm{~W} /(\mathrm{m})$ \\
\hline Material density & $\rho_{N a}=971 \mathrm{~kg} / \mathrm{m}^{3}$ & $\rho_{K}=862 \mathrm{~kg} / \mathrm{m}^{3}$ \\
\hline
\end{tabular}

After finishing the proposal of ion channels, the properties of used LF EMF were set up. The homogeneous electromagnetic field is generated by Helmholtz coils with a 1A current, frequency of $50 \mathrm{~Hz}$ and default sinusoidal excitation signal. This external field was then applied on the cell model. The results are compared with the simulations from [2], what can be seen in Figs. 4 and 5.

\footnotetext{
${ }^{1)}$ source: Chemicool - sodium, potassium, [online]: http://www.chemicool.com/elements
} 
a)

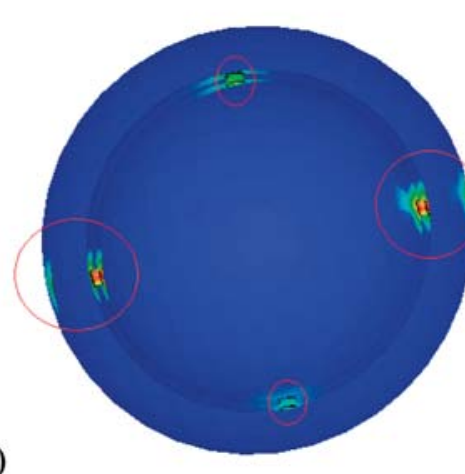

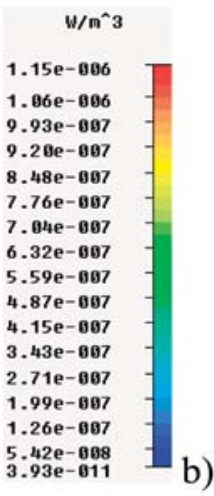

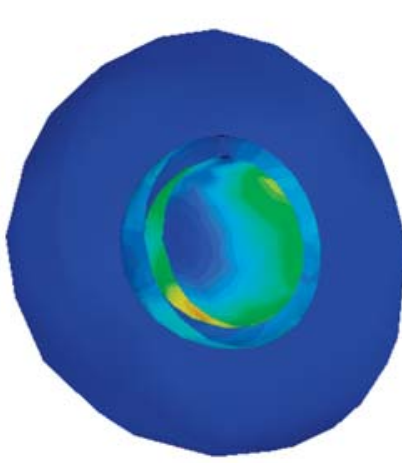

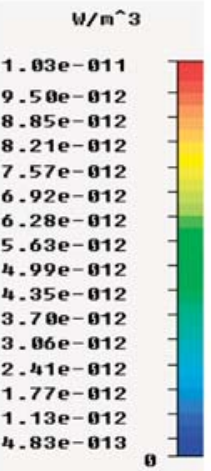

Fig. 4: Simulation results: a) Cross-section of El. Loss. Density around the ion channels in the cell membrane; b) Cross-section of El. Loss. Density without the channels in the membrane

a)

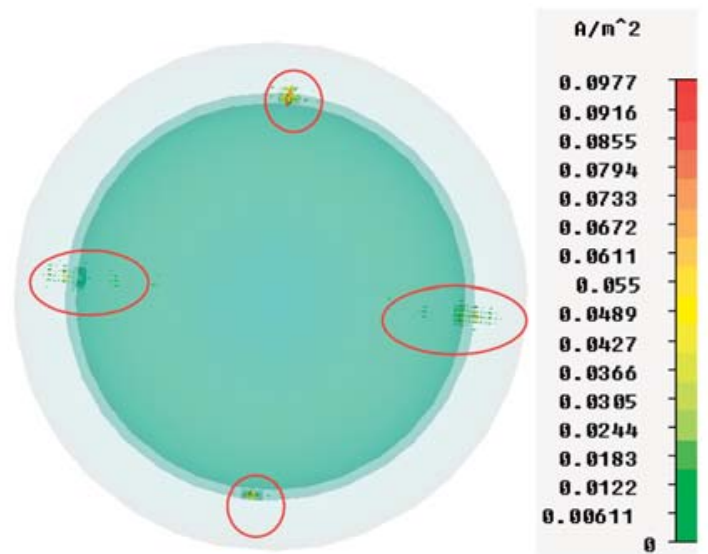

b)

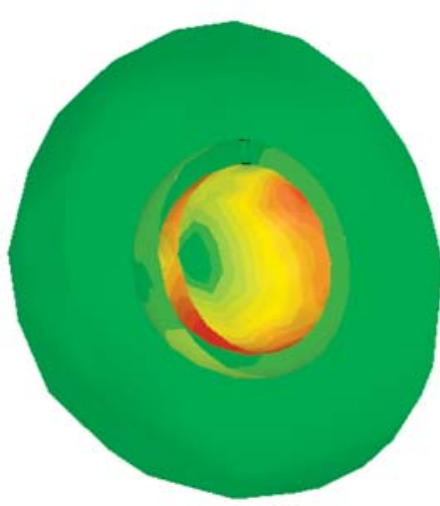

$A / m^{\wedge} 2$

3.36e-9อ6

$2.99 \mathrm{e}-986$

$2.79 \mathrm{e}-606$

$2.58 \mathrm{e}-096$

$2.38 \mathrm{e}-036$

$2.17 \mathrm{e}-606$

$1.96 \mathrm{e}-006$

$1.76 \mathrm{e}-006$

$1.55 \mathrm{e}-006$

1.34e-006

1.14e-006

9.29e- 997

$7.23 \mathrm{e}-097$

5.16e-697

3.16e- 697

Fig. 5: Simulation results: a) Eddy current distribution through the ion channels;

b) Eddy current distribution without the ion channels presence in the membrane

The differences between electric loss density of ion channels and the cell without ion channels exceed one order of magnitude. Similar situation is with eddy current distribution through the ion channels and without the ion channels. From the presented results it is our assumption that ion channels are probably the most influenced structures of the cell exposed to the external electromagnetic field and thus the changes on the ion channels level should have impact to the cell dividing processes as it is described in [7, 8].

The simulations further approve that not only electromagnetic properties, but also position (in this case rotation) of the cell in the electromagnetic field is important for evaluation of the effect on ion channels. As it is shown in Figs. 4 and 5 the channels near the horizontal axis where is the highest concentration of eddy currents are more influenced than the channels around the vertical axis.

\section{Conclusion}

It is well known that electromagnetic field could influence a living organism. Specific effects of electromagnetic field are quite well described and a lot of studies approved e.g. thermal impact of mobile phones to humans head structures [10]. In this article an attempt to describe some nonspecific effects of low frequency electromagnetic field was made.

For this purpose two main types of ion channels $-\mathrm{Na}^{+}$and $\mathrm{K}^{+}$channels were simulated (their importance is described in [7, $8]$ ). Based on our simulations it is probable that application of external low frequency electromagnetic field could influence the activation/inhibition of ion channels and thus could have impact on the transmembrane voltage and, finally, on cell proliferation.

It should be remembered that ion channels designed in this case represented only linear part of the whole problematic due to the simulations limitations which do not allow solution of such a complicated nonlinear problem as the case of biological cell is. To achieve the best simulation result as possible it is necessary to find solution in simulation of the nonlinear and linear structures together, which is currently a challenging task for future research. 


\section{References}

[1] CONE Jr., C.D.: Unified Theory on the Basic Mechanism of Normal Mitotic Control and Oncogenesis. .J. of Theoretical Biology, Vol. 30, 1971, pp. 151-181

[2] BARABAS, J., RADIL, R.: Investigation of Cellular Transmembrane Potential Variations via Exogenous Low Frequency Electromagnetic Fields. Clinician and Technology J., Vol. 40, 2010, pp. 41-45

[3] BINGGELI, R., WEINSTEIN, R. C.: Membrane Potentials and Sodium Channels: Hypotheses for Ggrowth Regulation and Cancer Formation Based on Changes in Sodium Channels Gap Junctions. J. of Theoretical Biology. Vol. 123, 1986, pp. 377-401

[4] SIMURDA, J.: Bioelectrical Phenomena I, CERM Brno, 1995

[5] GIMSA, J., WACHNER, D. A.: Unified Resistor-capacitor Model for Impedance, Dielectrophoresis, Electrorotation, and Induced Transmembrane Potential. Biophysical J., 1998, vol. 75, No. 1, pp. 1107-1116.

[6] KUNZELMANN, K.: Ion channels and cancer. The J. of Membrane Biology, Vol. 205, 2005, pp. 159-173

[7] ROGER, S., POTIER, M., VANDIER, CH., BESSON, P., LE GUENNEC, J-Y.: Voltage-Gated Sodium Channels: New Targets in Cancer Therapy? Current Pharmaceutical Design, 12, 2006, pp. 3681-3695

[8] PSENAKOVA, Z., BENOVA, M.: Measurement evaluation of EMF effect by mobile phone on human head phantom. Advances in Electrical and Electronic Engineering, Vol. 7, 2008, pp. 346-349. 